\title{
Autogenous bone particles combined with platelet-rich plasma can stimulate bone regeneration in rabbits
}

\author{
HUANXIN XIE, LEI CAO, LINLIN YE, JUBAO DU, GUIXIANG SHAN, \\ JIE HU, CHUNJING JIANG and WEIQUN SONG
}

Department of Rehabilitation, Xuanwu Hospital, Capital Medical University, Beijing 100053, P.R. China

Received January 15, 2020; Accepted August 19, 2020

DOI: 10.3892/etm.2020.9409

\begin{abstract}
Long-term bone defects are a key clinical problem. Autogenous bone graft remains the gold standard for the treatment of these defects; however, improving the osteogenic properties and reducing the amount of autogenous bone is challenging. Autologous platelet-rich plasma (PRP) has been widely considered for treatment, due to its potentially beneficial effect on bone regeneration and vascularization. The aim of the present study was to explore the effects of autogenous bone particles combined with PRP on repairing segmental bone defects in rabbits. Briefly, a critical-size diaphyseal radius defect was established in 45 New Zealand White rabbits. Animals were randomly divided into four groups, according to the different implants: Group A, empty bone defect; group B, PRP; group C, autogenous bone particles + bone mesenchymal stem cells (BMSCs) on the left radius; group D, autogenous bone particles + PRP + BMSCs on the right radius. Bone samples were collected and further analyzed using $\mathrm{X}$-ray, histology and histomorphometry 4, 8 and 12 weeks post-surgery. In addition, the effect of PRP on cell proliferation was detected by Cell Counting Kit- 8 and the concentrations of growth factors (GFs), transforming GF (TGF)- $\beta 1$ and platelet-derived GF (PDGF), in PRP were verified by ELISA. $\mathrm{X}$-ray, histology and histomorphometry data revealed that the fraction area of the newly formed bone was larger in group D. In addition, PRP could improve cell proliferation, osteogenic differentiation and the release of GFs, TGF- $\beta 1$ and PDGF-AB. In conclusion, these findings indicated that an autogenous bone
\end{abstract}

Correspondence to: Professor Weiqun Song, Department of Rehabilitation, Xuanwu Hospital, Capital Medical University, 45 Changchun Street, Beijing 100053, P.R. China

E-mail: songwq66@126.com

Abbreviations: PRP, platelet-rich plasma; BMSCs, bone mesenchymal stem cells; H\&E, hematoxylin and eosin; ASCs, adipose-derived stem cells; MSCs, mesenchymal stem cells

Key words: autogenous bone particles, bone regeneration, PRP, BMSCs, bone defect particle + PRP + BMSC scaffold may be used as a potential treatment strategy for segmental defects in humans.

\section{Introduction}

The treatment of long-term bone defects represents a clinical challenge for orthopedic specialists (1). Bone regeneration and healing processes are regulated by three key factors: Scaffold materials, growth factors (GFs) and seeding cells (2). GFs have an important biochemical role in bone regeneration (3).

Platelet-rich plasma (PRP) is a concentration of platelets from the peripheral blood, containing $~ 300$ bioactive cytokines that, when released, can promote tissue repair (4). PRP contains a number of GFs (4). These activated factors are essential for tissue regeneration and wound healing (5). In 1987, PRP was first used for heart surgery (6) and in 1998, it was used in the initial stage of enhanced fracture healing (7). Since then, PRP has been widely used in orthopedics to promote bone regeneration $(8,9)$. Usually, $\mathrm{PRP}$ is administered at a concentration 3-5x higher than the baseline. It has been reported that concentrations of platelets and available cytokines involved in stimulating and accelerating the repair process are positively correlated (10). However, studies on PRP have yielded contradictory results, which is probably due to different PRP preparation techniques (11). Currently, there is no international standard protocol for the preparation of PRP (12). The present study protocol was adapted from Landesberg et al (13), which is able to maintain the content of GFs, platelet-derived GF (PDGF) and transforming GF (TGF)- $\beta$, as much as possible and has good osteogenic properties.

Bone grafting materials, such as autografts, allografts, xenografts and synthetic biomaterials, have been extensively studied $(2,14)$. Although bone substitute materials exhibit good performance (biological function, mechanical qualities, efficiency or safety), autologous bone is considered the gold standard for grafting materials (15). Wang et al (16) indicated that particulate bone powder grafts may accelerate bone defect healing, as compared with large bone grafts, in a rat radial defect model. Furthermore, our previous study suggested that autologous bone particle/titanium fiber composites improved the mechanical strength to some extent in a segmental bone defect model (17). Autologous bone particles (diameter, $300-500 \mu \mathrm{m}$ ) are able to maximize the number of bone cells that release different types of GFs, thus enhancing the contact 
area and the supplement of nutrients to the tissue. However, insufficient strength and donor unavailability have been identified as weaknesses $(18,19)$. Bone mesenchymal stem cells (BMSCs) are a type of pluripotent stem cell, which have been successfully used to reconstruct bone defects in clinical trials $(20,21)$.

The present study hypothesized that PRP may release GFs and promote osteogenesis. The aim of the current study was to explore a novel method of bone defect reconstruction and the mechanism underlying the effects of PRP on supporting bone formation.

\section{Materials and methods}

BMSC isolation and culture. New Zealand white rabbits (male; age, 6-9 months; weight, 2.5-3.0 kg) were obtained from Beijing Vital River Laboratory Animal Technology Co., Ltd. and were housed in the Animal Laboratory of Xuanwu Hospital (Beijing, China). All animals were kept in separate cages and housed under the following conditions: Temperature, $22 \pm 1^{\circ} \mathrm{C}$; relative humidity, $50 \pm 1 \%$ and on a 12 -h light/dark cycle. Food and water access was ad libitum. All animal studies, including the rabbit euthanasia procedure, were performed in compliance with the American Association for Accreditation of Laboratory Animal Care and Institutional Animal Care and Use Committee (IACUC) guidelines. All animal studies were approved by the IACUC of Capital Medical University (Beijing, China; ethics approval no. XWH2018070011). BMSCs were obtained as previously described $(17,22)$.

BMSCs were collected from the bilateral femurs of rabbits (22). Under sterile conditions, the bone marrow was collected and washed with Dulbecco's modified Eagle's medium (DMEM; Gibco; Thermo Fisher Scientific, Inc.). To harvest a single cell suspension containing BMSCs, the cell suspension was centrifuged for $5 \mathrm{~min}$ at $250 \mathrm{xg}$ and room temperature and resuspended with DMEM. Subsequently, the cell suspension $(5 \mathrm{ml})$ was slowly transferred to a $15 \mathrm{ml}$ centrifuge tube containing Ficoll-Paque $(5 \mathrm{ml}$ ) (Sigma-Aldrich; Merck $\mathrm{KGaA}$ ), and then centrifuged at $400 \mathrm{x} \mathrm{g}$ at room temperature for $25 \mathrm{~min}$. Isolated BMSCs were collected from the interphase (cloud-like cell layer) and washed with PBS. The cell-PBS mixture was centrifuged for $5 \mathrm{~min}$ at $250 \mathrm{xg}$ at room temperature and the supernatant was removed to obtain the isolated BMSCs. Isolated BMSCs were then cultured in DMEM containing 10\% fetal bovine serum (FBS) (Gibco; Thermo Fisher Scientific, Inc.) and 1\% penicillin-streptomycin solution (10 kU/ml 100X penicillin; $10 \mathrm{mg} / \mathrm{ml}$ streptomycin) in a humidified atmosphere containing $5 \% \mathrm{CO}_{2} / 95 \%$ air at $37^{\circ} \mathrm{C}$. Cells were cultured at a concentration of $1 \times 10^{6} / 1$ in a $25-\mathrm{cm}^{2}$ flask. The culture medium was changed three times per week. All experiments were performed using cells at passages 3-5.

PRP preparation. A total of $10 \mathrm{ml}$ whole blood was collected from each animal (same rabbits used for BMSC extraction) ear arteries. The blood then underwent a two-step centrifugation process [initially, blood was centrifuged at $200 \mathrm{x}$ g for $10 \mathrm{~min}$ at $20^{\circ} \mathrm{C}$ to remove red blood cells (bottom fraction), and then at $200 \mathrm{x} \mathrm{g}$ for $10 \mathrm{~min}$ at $20^{\circ} \mathrm{C}$ to obtain the PRP] [pre-activated PRP; (middle fraction, $1 \mathrm{ml}$; Fig. 1A and B)].
The mean platelet number was $10.77 \times 10^{8} / \mathrm{ml}\left(\mathrm{SD} 0.85 \times 10^{8}\right)$ and that of whole blood was $2.92 \times 10^{8} / \mathrm{ml}\left(\mathrm{SD} 0.28 \times 10^{8}\right)$. Thrombin activators [500 units bovine thrombin; cat. no. 0219990701; MP Biomedicals (Shanghai) Co., Ltd.in $1 \mathrm{ml} \mathrm{10 \%} \mathrm{calcium}$ chloride] were added to the pre-activated PRP and gently mixed to activate PRP (post-activated PRP). A total of $1 \mathrm{ml}$ activated PRP was then mixed with $1 \times 10^{7}$ BMSCs.

Animal model and grouping. A rabbit radial defect model was established bilaterally, as previously described (1). Briefly, 45 New Zealand male rabbits (male; age, 6-9 months; weight, 2.5-3.0 kg) were used. Rabbits obtained from the same company and housed under the same conditions as previously mentioned. Rabbits were anesthetized with intravenous $3 \%$ sodium pentobarbital $(30 \mathrm{mg} / \mathrm{kg}$ and were euthanized after surgery at 4,8 and 12 weeks by intravenous injection of $3 \%$ sodium pentobarbital sodium $(100 \mathrm{mg} / \mathrm{kg})$. The methods used to verify death included the lack of a heartbeat, no autonomous respiration for 2-3 min and no blink reflex. The iliac bone $(400 \mathrm{mg})$, stripped of the periosteum and cartilage, was obtained from each rabbit. After removing the bone marrow and endosteum, the iliac bone was ground into bone powder grafts of 300-500 $\mu \mathrm{m}$ in diameter using a spherical grinding drill in saline. Bone particles and PRP/BMSCs were mixed. Subsequently, the autogenous bone particle/PRP/BMSC compound was shaped and prepared for the next step (Fig. 1C and D). A $15-\mathrm{mm}$ segment of the bilateral radius defect was cut off in the middle of the radius. The defect site was then washed with physiological saline and the graft was fixed into the bone. Finally, muscles and skin were respectively sutured.

The rabbits were divided into the following groups: Group A, empty bone defect ( $\mathrm{n}=9)$; group B, bone defect filled with PRP ( $\mathrm{n}=9)$; group $\mathrm{C}$, bone defect filled with autogenous bone particles $(200 \mathrm{mg})+$ BMSCs $\left(1 \times 10^{7}\right)$ on the left radius; and group $\mathrm{D}$, bone defect filled with autogenous bone particles $(200 \mathrm{mg})+$ PRP $(0.5 \mathrm{ml})+$ BMSCs $\left(1 \times 10^{7}\right)$ on the right radius. There were 27 rabbits in group C (left) and D (right). After surgery, the rabbits were maintained in individual cages and were fed normally. Bone specimens were harvested 4, 8 and 12 weeks post-surgery.

Macroscopic observations. After euthanasia, defective radius specimens were dissected and images were captured to observe the following: i) Bone formation in the bone defect area; ii) osteogenesis and inflammatory reaction in the local area; and iii) the relationship between the surrounding soft tissue and the bone defect (8).

$X$-rayexamination and scoring. An X-ray device $(45 \mathrm{kV}, 100 \mathrm{~mA}$, $0.12 \mathrm{sec}$; Siemens 1350; Siemens AG) was used to investigate the bone-healing process. X-ray scoring was performed according to the Lane-Sandhu standards (Table I) (23), including bone connection, recanalization of the medullary cavity and bone formation in the bone defect area, which were scored by three independent examiners. The images were captured three per group at three time points. The Lane-Sandhu score of each image was the sum of sub-parts of the scoring standard. The mean of the scores given by the three observers were accepted to be the final score (23). 
Table I. Lane-Sandhu radiographic scoring system.

\begin{tabular}{|c|c|c|}
\hline Variable & Description & Score \\
\hline \multirow[t]{5}{*}{ Degree of bone formation } & No new bone formed & 0 \\
\hline & The area of new bone accounts for $25 \%$ of the defect area & 1 \\
\hline & The area of new bone accounts for $50 \%$ of the defect area & 2 \\
\hline & The area of new bone accounts for $75 \%$ of the defect area & 3 \\
\hline & The area of new bone accounts for $100 \%$ of the defect area & 4 \\
\hline \multirow[t]{3}{*}{ Degree of union } & Fracture line is fully visible & 0 \\
\hline & Fracture line is partially visible & 2 \\
\hline & Fracture line is not visible & 4 \\
\hline \multirow[t]{3}{*}{ Degree of medullary cavity remodeling } & No sign of remodeling & 0 \\
\hline & Recanalization of medullary cavity & 2 \\
\hline & Cortical bone structure forms after recanalization of medullary cavity & 4 \\
\hline
\end{tabular}
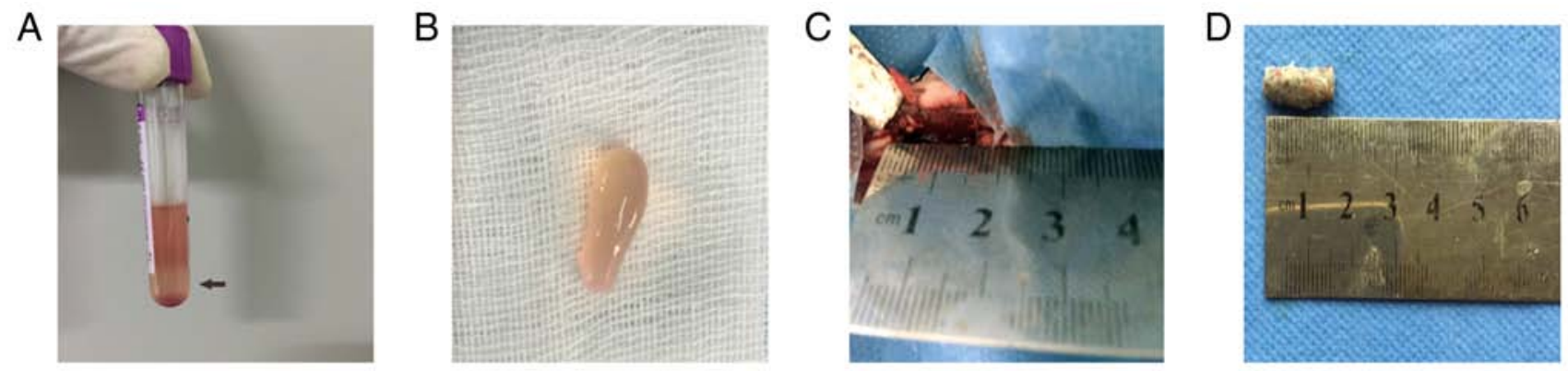

Figure 1. Materials and animal model. (A) PRP gel. (B) Gross appearance of the PRP scaffold. (C) Surgical induction of 15-mm bilateral defects in the radial bone. (D) Autogenous bone particle scaffold. PRP, platelet-rich plasma.

Histology and histomorphometry. Histological analysis of bone specimens was conducted using ImagePro software (version 7.0; Media Cybernetics, Inc.). Briefly, bone samples $(15-\mathrm{mm}$ new regenerated bone) were fixed in a $4 \%$ formaldehyde solution at room temperature for $48 \mathrm{~h}$ and rinsed with water. The samples were then dehydrated using graded alcohol and embedded in methyl methacrylate resin. Subsequently, sections $(5 \mu \mathrm{m})$ were stained with hematoxylin and eosin (H\&E) at room temperature for $5 \mathrm{~min}$ and examined under a light microscope (Nikon TE2000-U; Nikon Corporation). A single section from each animal was analyzed and 3 images in total were obtained from each group. Finally, the percentage of new bone in the defect area was calculated by histomorphometry using ImagePro image analysis software.

Immunohistochemistry. Sections prepared from groups C and $\mathrm{D}$ using the aforementioned process were analyzed by immunohistochemical staining. Sections $(5 \mu \mathrm{m})$ were deparaffinized in xylene and dehydrated with graded alcohol. Samples were then treated with 3\% hydrogen peroxide for $10 \mathrm{~min}$ and Tris/hydrochloric acid buffer for $5 \mathrm{~min}$ at room temperature, followed by incubation with an anti-osteocalcin monoclonal antibody (cat. no. ab13418; Abcam; 1:200) at $4^{\circ} \mathrm{C}$ overnight. Subsequently, sections were washed with PBS and incubated at room temperature with biotin-labeled goat anti-mouse IgG secondary antibodies (cat. no. A0286; Beyotime Institute of Biotechnology; 1:200) for $30 \mathrm{~min}$ and counterstained with hematoxylin (cat. no. H8070; Beijing Solarbio Science \& Technology Co., Ltd.) for $3 \mathrm{~min}$ at room temperature before observation using a light microscope. A single section from each animal was analyzed and 3 images in total were obtained from each group.

Cell proliferation assay. Two groups of cells were analyzed: i) BMSCs treated with DMEM $+10 \%$ FBS; and ii) BMSCs treated with DMEM + 10\% PRP for 7 days. Briefly, BMSCs $\left(100 \mu \mathrm{l} /\right.$ well, $\left.2 \times 10^{3}\right)$ were plated in 96 -well plates and incubated in an atmosphere containing $5 \% \mathrm{CO}_{2} / 90 \%$ air at $37^{\circ} \mathrm{C}$. At each time point (1-7 days), $10 \mu$ l Cell Counting Kit-8 (CCK-8) (cat. no. C0037; Beyotime Institute of Biotechnology) solution was added to each well and incubated for $2 \mathrm{~h}$ at $37^{\circ} \mathrm{C}$. The absorbance at $450 \mathrm{~nm}$ was determined using a microplate reader (SpectraMax 340PC; Molecular Devices, LLC).

ELISA. The concentrations of TGF- $\beta 1$ and PDGF-AB, in whole blood plasma, and pre- and post-activated PRP were detected using an TGF- $\beta 1$ ELISA kit (cat. no. SEKRT-0401; Beijing Solarbio Science \& Technology Co., Ltd.) and PDGF-AB ELISA kit (cat. no. SEKRT-0030-96T; Beijing Solarbio Science \& Technology Co., Ltd.), according to the manufacturer's protocol. Briefly, a $50 \mu \mathrm{l}$ sample was added to each well of 96-well microplates that were coated with a monoclonal antibody against TGF- $\beta 1$ or PDGF-AB for $2 \mathrm{~h}$ at $37^{\circ} \mathrm{C}$. 
After washing with buffer, each sample was incubated with horseradish peroxidase-conjugated cytokine for $1 \mathrm{~h}$. Samples were then incubated with substrate solution for $30 \mathrm{~min}$ at $37^{\circ} \mathrm{C}$ and stopped with termination solution. Results were obtained by measuring absorbance at $450 \mathrm{~nm}$ using a microplate reader.

Osteogenic differentiation of BMSCs. BMSCs were cultured in 24-well plates $\left(5 \times 10^{4} / \mathrm{cm}^{2}\right)$. When the cell density reached $60-70 \%$, cells were exposed to osteogenic induction medium (cat. no. RBXMX-90021; Cyagen Biosciences, Inc.), including $100 \mathrm{U} / \mathrm{ml}$ penicillin-streptomycin, $1 \%$ glutamine, $10 \mathrm{nmol} / \mathrm{l}$ dexamethasone, $0.2 \mathrm{mmol} / \mathrm{l}$ ascorbate and $10 \mathrm{mmol} / \mathrm{l}$ $\beta$-glycerophosphate. They were divided into two groups, one group was treated with $10 \%$ FBS and the other with $10 \%$ PRP at room temperature for 2 weeks. The medium was changed every 3 days. After 2 weeks, mineralization was detected by alkaline phosphatase (ALP) staining and Alizarin Red staining (ARS).

$A L P$ staining. After induction in osteogenic induction medium for 2 weeks, in vitro mineralization was assessed using an ALP staining kit (cat. no. C3026; Beyotime Institute of Biotechnology). Briefly, the cells were washed three times with PBS and fixed in $4 \%$ paraformaldehyde for $10 \mathrm{~min}$ at room temperature. Subsequently, the cells were embedded in ALP staining solution for $1 \mathrm{~h}$ at room temperature in a 24-well plate, followed by three PBS washes. A light microscope (Nikon TE2000-U) was then used to obtain the image.

$A R S$. An ARS kit, provided with the differentiation medium (cat. no. RBXMX-90021; Cyagen Biosciences, Inc.), was used to detect matrix mineralization depositions after 2 weeks. After washing three times with PBS, the cells were fixed with $4 \%$ formalin for $10 \mathrm{~min}$ at room temperature, and then stained with ARS solution for $30 \mathrm{~min}$. Subsequently, the solution was removed, the cells were rinsed three times with PBS and images ( $n=3$ per group) were captured under a light microscope (Nikon TE2000-U; Nikon Corporation).

ALP activity assay. BMSCs were treated with osteogenic induction media in 24-well plates for 2 weeks. They were then divided into the 10\% PRP and 10\% FBS groups. The cells were collected and the level of ALP activity was determined using an ALP activity kit (cat. no. P0321; Beyotime Institute of Biotechnology) according to manufacturer's protocol. The absorbance values of samples were measured at $405 \mathrm{~nm}$ using a spectrophotometer. The total protein content was determined via the BCA method using aliquots of the same samples with the protein assay kit (cat. no. P0010; Beyotime Institute of Biotechnology). ALP activity was normalized to total protein concentration. The formula for the ALP activity was as follows: ALP activity $(\mathrm{U} / \mathrm{g}$ prot $)=[($ absorbance of the determination tube/absorbance of the standard tube) $\mathrm{x}$ the amount of nitrophenol in the standard tube]/grams of total protein.

Statistical analysis. For continuous variables, data are expressed as the mean \pm SD. The cell proliferation assay data were analyzed using a two-way ANOVA followed by Bonferroni post hoc test. ELISA results were analyzed using one-way ANOVA followed by the least significant difference post hoc test. Histomorphometry and ALP activity were analyzed using an independent samples t-test between the two groups. Data analysis was performed using SPSS 22.0 software (IBM, Corp.) For categorical variables, data are expressed as a median (interquartile range). Lane-Sandhu score was analyzed using Kruskal-Wallis test with Dunn's post hoc test. Data analysis was performed using SAS 9.4 software (SAS, Inc.). $\mathrm{P}<0.05$ was considered to indicate a statistically significant difference.

\section{Results}

Macroscopic observations of bone defects with and without treatment. After surgery, the incisions to the experimental animals exhibited light swelling and there was no obvious secretion around the incisions. After $\sim 4$ days, the swelling gradually subsided, and the incision healed well in $\sim 12$ days. Images of the gross specimen of each group are shown in Fig. 2A. Group A did not exhibit complete repair of the bone defect after 4, 8 and 12 weeks. Surrounded by soft tissue, there was a small number of calluses at the end of the fracture at 4/8 weeks. At 12 weeks, bone sclerosis and enlargement were observed, the defects could be seen, and they were replaced by soft tissue. Group B was similar to group A. In groups $\mathrm{C}$ and $\mathrm{D}$, the defects were wrapped with dense fibrous tissue and new bone processes were observed at the edge of the bone defect. Some callus formation was identified. There was increased bone formation in group $\mathrm{D}$ in comparison to group C. At 8 weeks, continuous new bone was observed in the bone defect area of group $\mathrm{C}$, with slight depression at the radial side of the defect. The newly regenerated bone in the middle of the defect area was fused with the ulna. Bone in group D also formed continuously. The radial part of the defect was less depressed than that of group $\mathrm{C}$, and the new bone was also connected partly with the ulna. The bone defect area of group $\mathrm{C}$ at 12 weeks displayed continuous new bone formation, less depression in the radial part and nearly well-integrated shape. Group D formed bony connections at 12 weeks and had a well-integrated shape.

Radiographic evaluation. . As shown in Fig. 2B, there was no bony bridge in groups $\mathrm{C}$ and $\mathrm{D}$ at 4,8 , or 12 weeks. A few bone calluses were visible around the broken end in group A and B at 4 weeks. The broken end was ossified with a large fissure in the defect center and medullary cavities were blocked at 8 and 12 weeks. Bone healing in group D was superior to that in group $\mathrm{C}$ at three time points. At week 4 after surgery, bone union had occurred around the bone defect sites in groups $\mathrm{C}$ and $\mathrm{D}$. At 8 weeks, continuous bone callus formation and partial recanalization of the medullary cavity segment was observed in groups $\mathrm{C}$ and D. Remodeling was superior in group D to that in group C.

Lane-Sandhu radiographic score for the evaluation of defect repair revealed the following mean scores: No statistically significant differences were observed between groups $\mathrm{A}$ and $\mathrm{B}$ at each time point $(\mathrm{P}>0.05)$. Furthermore, a higher score was found in group D compared with the score in the other groups, and the difference was statistically significant at each time point $(\mathrm{P}<0.05$; Fig. $2 \mathrm{C}$; Table II). 
Table II. Lane-Sandhu radiographic scoring results.

\begin{tabular}{lccc}
\hline Group & 4 weeks & 8 weeks & 12 weeks \\
\hline A & $0.19(0.19-0.20)$ & $0.34(0.32-0.35)$ & $0.48(0.47-0.49)$ \\
B & $0.19(0.18-0.20)$ & $0.38(0.34-0.39)$ & $0.56(0.55-0.58)$ \\
C & $2.91(2.57-3.20)$ & $7.83(7.62-7.86)$ & $10.02(9.95-10.25)$ \\
D & $3.36(3.23-3.58)^{\mathrm{a}}$ & $8.61(8.37-8.75)^{\mathrm{a}}$ & $11.35(11.10-11.50)^{\mathrm{a}}$
\end{tabular}

Data are presented as median (interquartile range). Lane-Sandhu radiographic score of group D was higher than of the other groups ( ${ }^{a} \mathrm{P}<0.05$ ) Group A, empty bone defect; group B, PRP; group C, autogenous bone particles + BMSCs on the left radius; group D, autogenous bone particles + platelet-rich plasma + bonemesenchymal stem cells on the right radius.
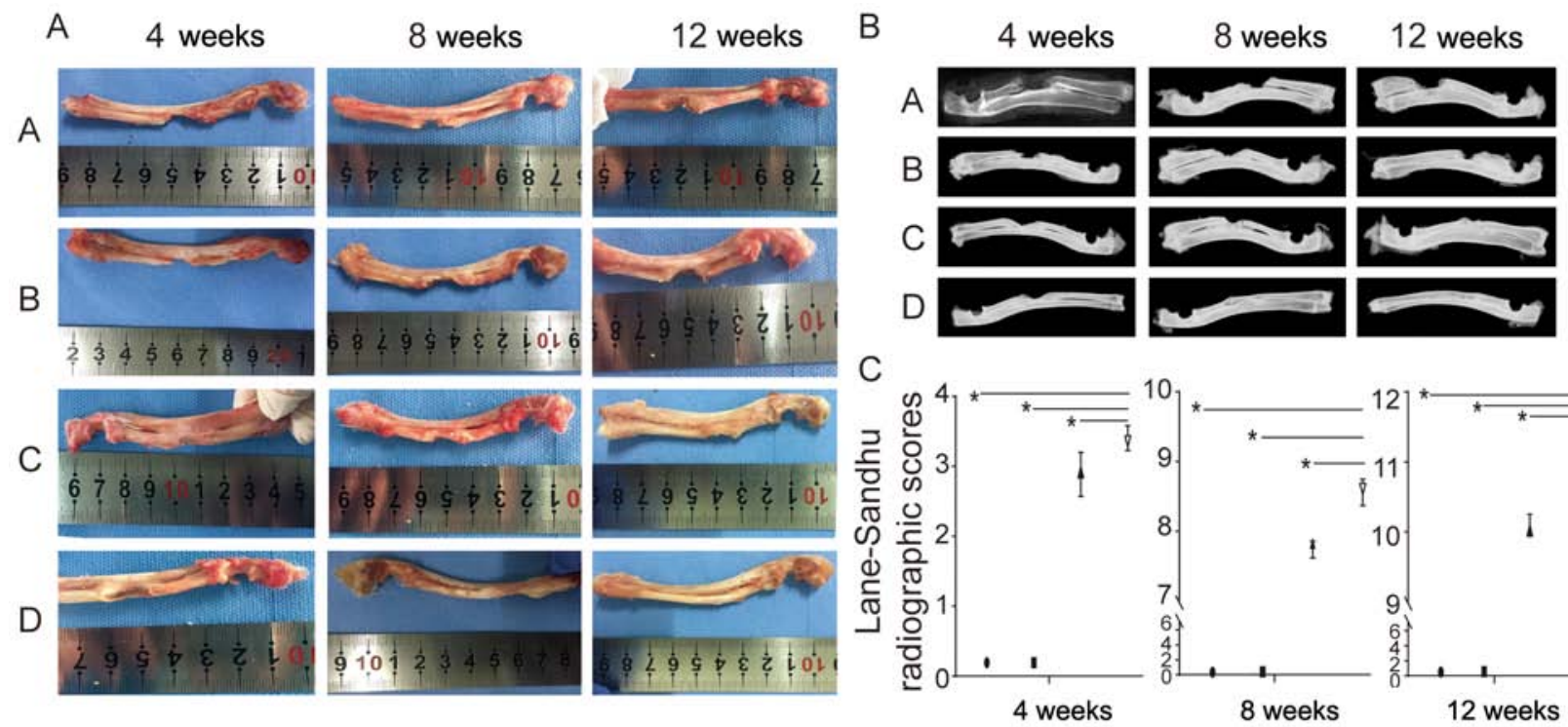

Figure 2. (A) In vivo macroscopic observation of groups A, B, C and D during osteogenic repair at three time points (4, 8 and 12 weeks). (B) Representative radiograph from groups $\mathrm{A}, \mathrm{B}, \mathrm{C}$ and $\mathrm{D}$ at three time points (4, 8 and 12 weeks). (C) Lane-Sandhu radiographic score of the four groups ( $\mathrm{n}=3$ /group). Group A, empty bone defect; group B, PRP; group C, autogenous bone particles + BMSCs on the left radius; group D, autogenous bone particles + PRP + BMSCs on the right radius. ${ }^{*} \mathrm{P}<0.05$. $\mathrm{PRP}$, platelet-rich plasma; BMSCs, bone mesenchymal stem cells.

Histology and histomorphometry. X-ray images revealed that there was no bridging and repair in groups A and B. Thus, histological data and histomorphometry were only obtained for groups C and D.

H\&E staining revealed that trabecular bone was disturbed at 4 weeks. The trabecular bone tended to be smooth, and partial bone marrow cavity re-opening was observed at 8 weeks. At 12 weeks, good bone reconstruction and medullary cavity recanalization was observed in groups $C$ and D (Fig. 3A and B). The trabecular bone was mature and intensive lamellar bone was generated. Furthermore, better bone formation was observed in group D compared with in group $\mathrm{C}$ at the three time points.

Histomorphometry was performed using ImagePro software; the results indicated that at 4 weeks the ratios of the fraction area of the newly formed bone for groups $\mathrm{C}$ and $\mathrm{D}$ were $8.35 \pm 0.52$ and $9.78 \pm 0.36$, respectively, and the difference was statistically significant $(\mathrm{P}<0.05$; Fig. 3C). Similarly, at 8 and 12 weeks, group D $(23.83 \pm 0.54$ and $42.60 \pm 1.47)$ exhibited a significantly increased ratio compared with that in group C (20.85 \pm 0.50 and $39.02 \pm 0.58 ; \mathrm{P}<0.05$; Fig. $3 C)$.
Immunohistochemistry. Immunohistochemical staining for osteocalcin was used to detect new bone specimens at three time points following surgery. Briefly, osteocalcin-positive cells were observed in the regeneration bone area of groups C and D throughout newly formed bone with lacunae. The area that stained positive for bone osteocalcin in group D was larger that in group $\mathrm{C}$ at each time point and bone formation in group D was increased compared to group C (Fig. 3D).

$C C K-8$ assay. To evaluate BMSC proliferation with or without PRP, growth curves were established using a CCK- 8 kit. The proliferative activity of the PRP group was increased compared with that of the control group (without PRP). The difference between the two groups was significant $(\mathrm{P}<0.05$; Fig. 4A).

GF levels in PRP. The concentrations of TGF- $\beta 1$ and PDGF-AB in the three groups (whole blood plasma, and pre-and post-activated PRP) were measured using ELISA. As shown in Fig. 4B, the levels of the two GFs were higher in post-activated PRP $(102.11 \pm 1.56$ and $72.27 \pm 1.37 \mathrm{ng} / \mathrm{ml})$ compared with those in pre-activated PRP $(26.35 \pm 0.40$ and 
A

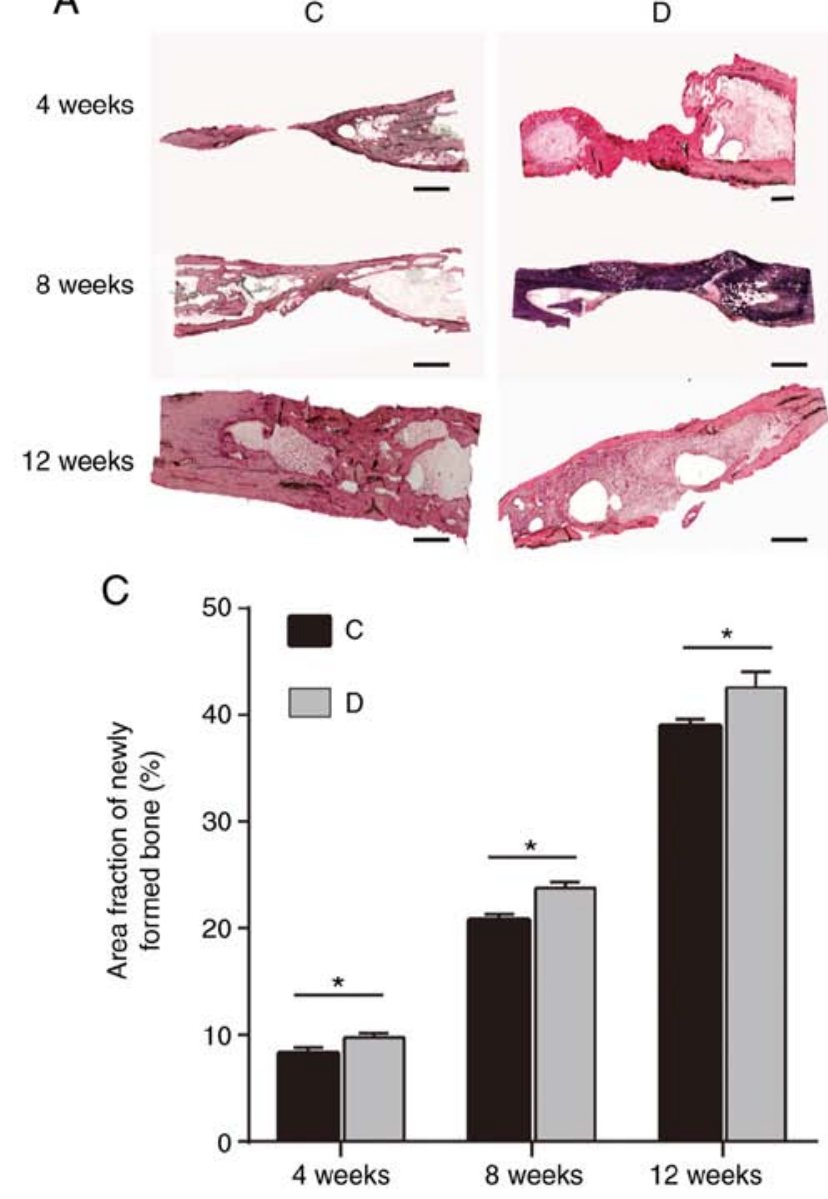

B

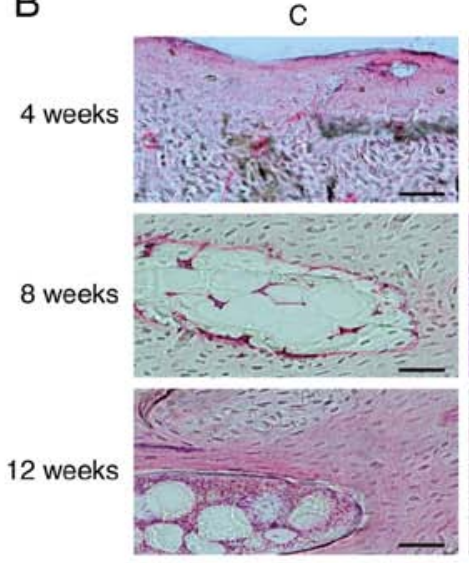

D

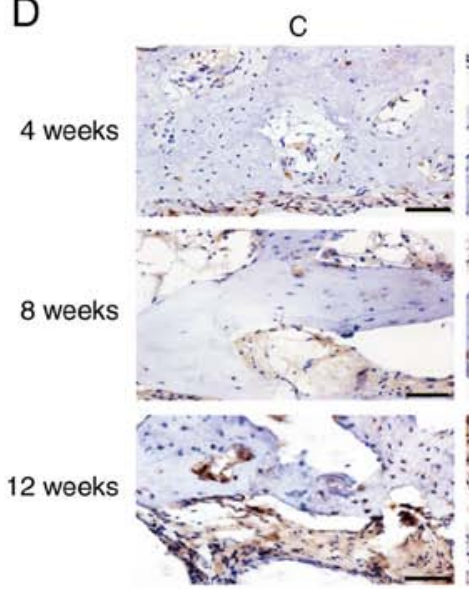

D

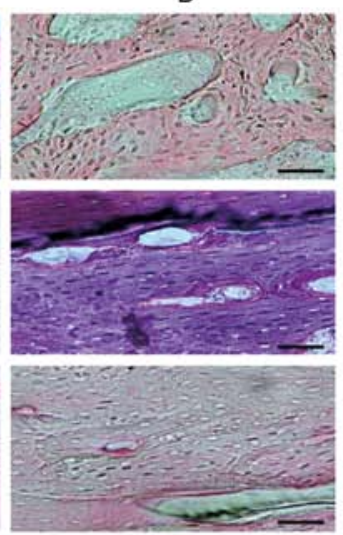

D

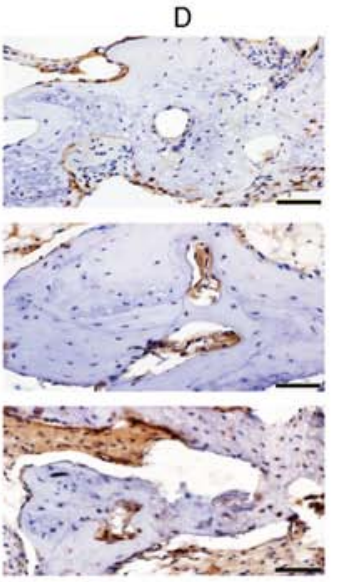

Figure 3. H\&E staining of rabbit radius samples at 4, 8 and 12 weeks from groups C and D. (A and B) H\&E staining. (A) Magnification, $\mathrm{x} 4$; scale bars, $3,000 \mu \mathrm{m}$. (B) Magnification, $\mathrm{x} 40$; scale bars, $100 \mu \mathrm{m}$. (C) Fraction area of the newly formed bone within the former defect as a percentage of total defect area at 4, 8 and 12 weeks (n=3/group). (D) Immunohistochemical observation of osteocalcin in groups $\mathrm{C}$ and $\mathrm{D}$ at 4,8 and 12 weeks. Scale bar, $100 \mu \mathrm{m}$. Group C, autogenous bone particles $+\mathrm{BMSCs}$ on the left radius; group $\mathrm{D}$, autogenous bone particles $+\mathrm{PRP}+\mathrm{BMSCs}$ on the right radius. ${ }^{*} \mathrm{P}<0.05$. H\&E, hematoxylin and eosin; PRP, platelet-rich plasma; BMSCs, bone mesenchymal stem cells.

$16.60 \pm 0.85 \mathrm{ng} / \mathrm{ml})$ and whole blood plasma $(30.57 \pm 0.78$ and $21.70 \pm 0.86 \mathrm{ng} / \mathrm{ml} ; \mathrm{P}<0.001)$.

Osteogenic effect of PRP on BMSCs. To detect the osteogenic potential of PRP, ALP and ARS staining were performed after 14 days of osteogenic induction. The results revealed that cells treated with $10 \%$ PRP exhibited more matrix mineralization compared with in cells treated with 10\% FBS (Fig. 4C). ALP activity was measured following osteogenic induction for 2 weeks. The ALP activity of BMSCs in the 10\% PRP group was higher compared with that in the $10 \%$ FBS group $(\mathrm{P}<0.001$; Fig. 4D).

\section{Discussion}

The present study revealed that the combination of PRP, BMSCs and autogenous bone particles stimulated bone regeneration. The results confirmed the hypothesis that PRP may be beneficial to bone grafting, as it had positive effects on cell proliferation and osteogenic differentiation, and, when activated, it induced the expression of TGF- $\beta 1$ and PDGF-AB. Fracture healing may be divided into three stages: i) Inflammatory phase (hematoma and granulation tissue), this stage lasts about 2-4 weeks. ii) Reparative phase (callus formation), in areas closer to the well-vascularized healthy bone tissue, osteogenic cells differentiate into osteoblasts, which produce spongy bone trabeculae, this stage lasts $\sim 4-8$ weeks. iii) Remodeling phase, this is the final phase of healing, where compact bone replaces spongy bone around the periphery of the fracture site, this stage lasts $\sim 8-12$ weeks (23). Therefore, 4,8 and 12 weeks were chosen as the time points assessed in the present study; this is consistent with other studies $(23,24)$.

Tajima et al (21) reported that adipose-derived stem cells (ASCs) combined with PRP exhibited an augmentative effect on bone regeneration in a rat calvarial defect model. Moreover, Blaszczyk et al (25) suggested that a combination of poly(L/DL-lactide) $80 / 20$ and PRP may promote bone regeneration in a large sheep calvarial defect. Furthermore, Qi et al (26) proposed the use of BMSC/PRP gel/calcium phosphate particles as a treatment strategy for bone defect repair in patients. Notably, the present study revealed that autogenous bone particles/PRP/BMSCs had a good effect on connecting bone defects. Nevertheless, certain studies have reported some contradictory results in the use of PRP for bone defect healing. For example, Guerra et al (27) 

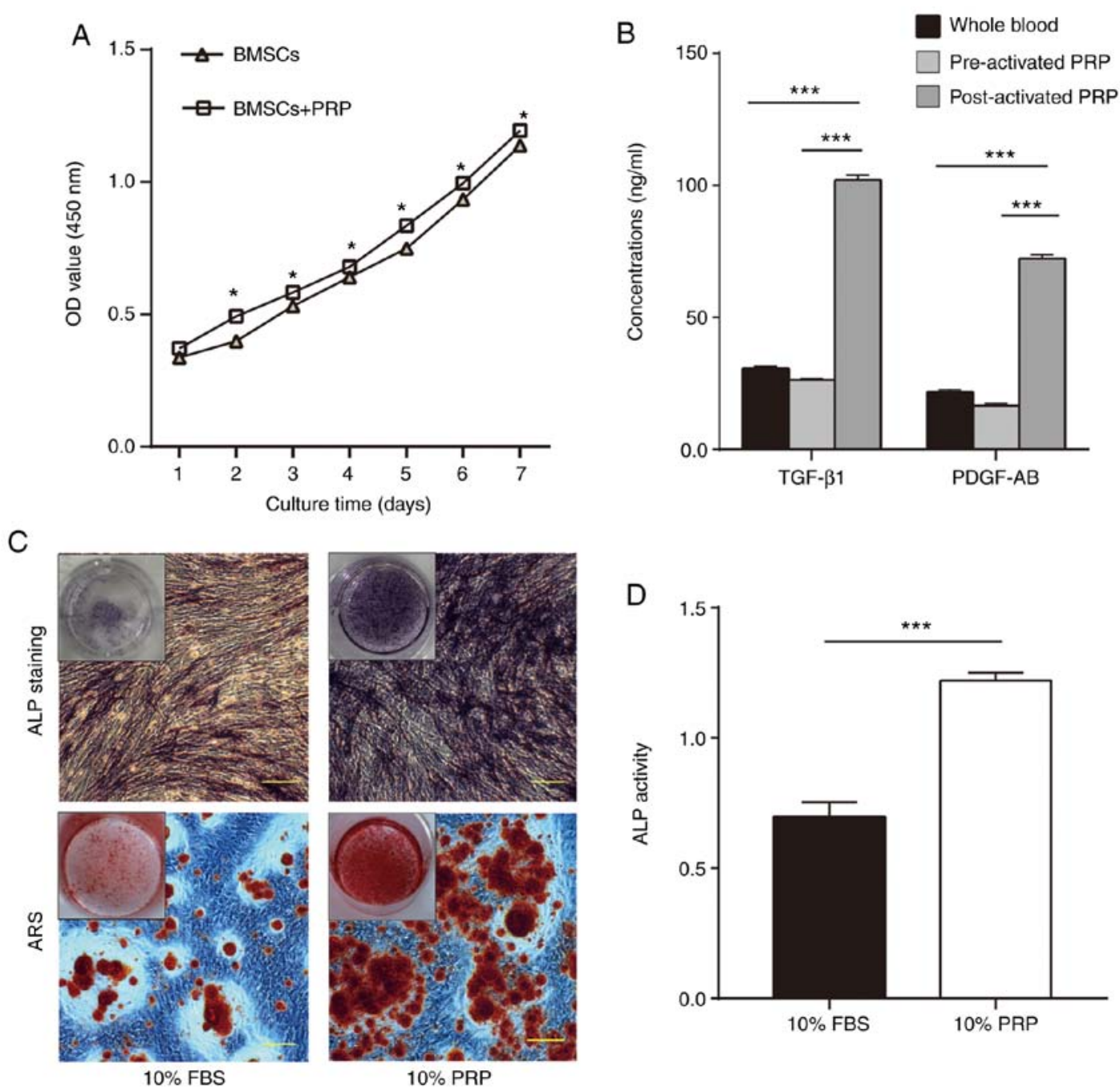

Figure 4. Effects of PRP on BMSCs and growth factors in PRP. (A) Growth curve of BMSCs and BMSCs treated with PRP. (B) Concentrations of growth factors secreted from pre- and post-activated PRP, and whole blood plasma. (n=5/group). (C) ALP staining and ARS were performed to indicate mineral deposition. Scale bars, $100 \mu \mathrm{m}$. (D) ALP activity was measured during osteogenic differentiation (n=3/group)." $\mathrm{P}<0.05$ and ${ }^{* * *} \mathrm{P}<0.001$. BMSCs, bone mesenchymal stem cells; ALP, alkaline phosphatase; ARS, Alizarin Red staining; PRP, platelet-rich plasma; OD, optical density; FBS, fetal bovine serum; PRP, platelet-rich plasma.

examined the femur defect-healing effect after treating rabbits with $\mathrm{NuOss}^{\mathrm{TM}}$, a commercially available self-expanding composite graft, combined with plasma rich in GFs (PRGF), and $\mathrm{NuOss}^{\mathrm{TM}}$ with a resorbable collagen membrane (RCM). Their data suggested that the percentage of bone tissue in direct contact with the implant surface was significantly lower in the NuOss ${ }^{\mathrm{TM}}+$ PRGF group compared with that in the $\mathrm{NuOss}^{\mathrm{TM}}+\mathrm{RCM}$ group at 4 and 8 weeks after surgery. Another study demonstrated that there was no statistical difference in the total new bone formation at an early stage ( $<4$ weeks post-surgery/treatment) in a rabbit calvarial defect treated with PRP mixed with particulate autogenous bone and autogenous bone alone (28). These contradictory results could be explained by the use of different methods for PRP preparation, as there is currently no universal standard for it.

The methods for PRP activation may also indirectly influence the concentration of GFs. Landesberg et al (13) suggested that platelets could be destroyed by centrifugation at $250 \mathrm{x} \mathrm{g}$, thus causing a further decrease in the release of GFs. In the previous study by Guerra et al (27), low bone formation was observed following centrifugation at $391 \mathrm{x}$. The protocol followed by Landesberg et al (13) is considered more conducive to releasing osteogenic GFs and promoting osteogenesis. Therefore, the present study adapted the protocol followed by Landesberg et al (13). Currently, the hybrid application of calcium chloride and thrombin in chitosan is the most commonly used approach to activate PRP compared with calcium chloride, thrombin or chitosan alone. The method of hybrid application of calcium chloride and thrombin to activate PRP followed by the Landesberg protocol was chosen in the present study. Finally, the physiological state and living environment of the animals varied, and different animals and species could have a vital effect on the ability of PRP.

Recently, autologous PRP has been successfully applied as 3-D scaffold material in rebuilding human cartilage (29). In addition, Tajima et al (21) transplanted a mixture of ASCs and PRP into the calvarial defect of rats and found that the mixture induced defect healing. Nevertheless, other studies have suggested that PRP has no effect on osteochondral defects of the talus (30). Guerra et al (27) indicated that PRP might 
induce slight bone formation in calvarial defects. The present results were in line with data obtained by Guerra et al (27). In the present study, radial defects in rabbits were filled with BMSCs and PRP (group B) and slight bone formation was observed, with no bridge formation around the defect. Previous research examined bone defects, of different types and sizes $(21,27,29,30)$. In small defects or cartilage injury, PRP alone performed well in remodeling and repair, whereas in the large and segmental bone defects, PRP scaffold had limitations in osteogenesis $(21,27,29,30)$.

Following $\mathrm{X}$-ray examination in vivo, as well as $\mathrm{H} \& \mathrm{E}$ staining and histomorphometry ex vivo, it was revealed that rabbits treated with autogenous bone particles combined with PRP and BMSCs exhibited improved bone healing compared with that in rabbits treated with autogenous bone particles and BMSCs. A combination of autogenous bone particles with PRP and BMSCs was conducive to bone bridge reconstruction through the proliferation of BMSCs and the release of GFs by platelets in PRP. In addition, the present study demonstrated that BMSCs treated with PRP had a greater proliferative activity compared with that of BMSCs without PRP. Certain studies had similar outcomes, indicating that PRP could be used to induce MSC proliferation and promote bone formation $(31,32)$. The present results revealed that treatment with 10\% PRP indicated stronger ALP and Alizarin Red staining compared with that in cells treated with $10 \%$ FBS. PRP exhibited a good osteogenic differentiation ability. The present study also showed that post-activated PRP contained higher concentrations of GFs, TGF- $\beta 1$ and PDGF-AB, than pre-activated PRP. It has been reported that PRP could improve bone healing or bone remodeling, due to the GFs release from PRP. PRP contains several GFs, TGF- $\beta 1$ and PDGF-AB being the most prominent (12). TGF- $\beta 1$ may stimulate osteoblastic and fibroblastic properties, and the mitogenic effects of other GFs, and contribute to BMSC differentiation. PDGF-AB is responsible for cell proliferation, and guiding cell migration, blood vessel repair and regeneration $(33,34)$. The release of GFs may be the reason why PRP promotes cell proliferation and osteogenic differentiation, as well as osteogenesis of bone defects in vivo. Autologous bone particles can preserve BMSCs and enhance the bone contact area, whereas PRP can increase cell proliferation and osteogenic differentiation, and release GFs, thus supplying more nutrients to the surrounding tissues.

There were two limitations to the present study. Firstly, this study did not use various doses of PRP to verify osteogenic capacity; only a recommended dose, according to the literature (13), was used. Secondly, it was shown that PRP could promote bone particle formation; however, the mechanism underlying bone regeneration remains unclear. Future studies should focus on estimating the ability of osteogenesis for different preparation methods, doses and centrifugation speeds of PRP, and should explore the osteogenic potential of BMSCs.

In conclusion, PRP itself could not bridge the defect. The combination of particulate autologous bone grafts and PRP could further improve bone healing in a diaphyseal rabbit model compared with the particulate autologous bone group. The release of cytokines by platelets in PRP may have an important role in bone repair. This study supported the autologous use of PRP for bone healing as a treatment option forlong-term bone defects.

\section{Acknowledgements}

Not applicable.

\section{Funding}

No funding was received.

\section{Availability of data and materials}

The datasets used and/or analyzed during the current study are available from the corresponding author on reasonable request.

\section{Authors' contributions}

HX and LC carried out the experiments and drafted the manuscript. WS, LY, CJ and JH performed the statistical analysis and participated in the study design. GS and JD helped to collect data and performed the statistical analysis. All authors read and approved the final manuscript.

\section{Ethics approval and consent to participate}

All animal studies, including the rabbit euthanasia procedure, were approved by the IACUC of Capital Medical University, and were conducted according to the American Association for Accreditation of Laboratory Animal Care and the IACUC guidelines.

\section{Patient consent for publication}

Not applicable.

\section{Competing interests}

The authors declare that they have no competing interests.

\section{References}

1. Kasten P, Vogel J, Geiger F, Niemeyer P, Luginbühl R and Szalay K: The effect of platelet-rich plasma on healing in critical-size long-bone defects. Biomaterials 29: 3983-3992, 2008.

2. Nooeaid P, Salih V, Beier JP and Boccaccini AR: Osteochondral tissue engineering: Scaffolds, stem cells and applications. J Cell Mol Med 16: 2247-2270, 2012.

3. Cheng X, Wan Q and Pei X: Graphene Family Materials in Bone Tissue Regeneration: Perspectives and Challenges. Nanoscale Res Lett 13: 289, 2018.

4. Liao HT, Tsai MJ, Brahmayya M and Chen JP: Bone Regeneration Using Adipose-Derived Stem Cells in Injectable Thermo-Gelling Hydrogel Scaffold Containing Platelet-Rich Plasma and Biphasic Calcium Phosphate. Int J Mol Sci 19: 2537, 2018.

5. Wen Y, Gu W, Cui J, Yu M, Zhang Y, Tang C, Yang P and Xu X: Platelet-rich plasma enhanced umbilical cord mesenchymal stem cells-based bone tissue regeneration. Arch Oral Biol 59: 1146-1154, 2014

6. Ferrari M,Zia S, Valbonesi M, Henriquet F, Venere G, Spagnolo S, Grasso MA and Panzani I: A new technique for hemodilution, preparation of autologous platelet-rich plasma and intraoperative blood salvage in cardiac surgery. Int J Artif Organs 10: 47-50, 1987.

7. Marx RE, Carlson ER, Eichstaedt RM, Schimmele SR, Strauss JE and Georgeff KR: Platelet-rich plasma: Growth factor enhancement for bone grafts. Oral Surg Oral Med Oral Pathol Oral Radiol Endod 85: 638-646, 1998. 
8. Xie X, Wang Y, Zhao C, Guo S, Liu S, Jia W, Tuan RS and Zhang C: Comparative evaluation of MSCs from bone marrow and adipose tissue seeded in PRP-derived scaffold for cartilage regeneration. Biomaterials 33: 7008-7018, 2012.

9. Son SR, Sarkar SK, Nguyen-Thuy BL, Padalhin AR, Kim BR, Jung HI and Lee BT: Platelet-rich plasma encapsulation in hyaluronic acid/gelatin-BCP hydrogel for growth factor delivery in BCP sponge scaffold for bone regeneration. J Biomater Appl 29: 988-1002, 2015

10. Zou J, Yuan C, Wu C, Cao C and Yang H: The effects of platelet-rich plasma on the osteogenic induction of bone marrow mesenchymal stem cells. Connect Tissue Res 55: 304-309, 2014.

11. Chahla J, Cinque ME, Piuzzi NS, Mannava S, Geeslin AG, Murray IR, Dornan GJ, Muschler GF and LaPrade RF: A Call for Standardization in Platelet-Rich Plasma Preparation Protocols and Composition Reporting: A Systematic Review of the Clinical Orthopaedic Literature. J Bone Joint Surg Am 99: 1769-1779, 2017

12. Oryan A, Alidadi S and Moshiri A: Platelet-rich plasma for bone healing and regeneration. Expert Opin Biol Ther 16: 213-232, 2016.

13. Landesberg R, Burke A, Pinsky D, Katz R, Vo J, Eisig SB and $\mathrm{Lu} \mathrm{HH}$ : Activation of platelet-rich plasma using thrombin receptor agonist peptide. J Maxillofac Surg 63: 529-535, 2005

14. Oryan A, Alidadi S, Moshiri A and Maffulli N: Bone regenerative medicine: Classic options, novel strategies, and future directions. J Orthop Surg Res 9: 18, 2014.

15. Duan R, Barbieri D, de Groot F, de Bruijn JD and Yuan $\mathrm{H}$ Modulating Bone Regeneration in Rabbit Condyle Defects with Three Surface-Structured Tricalcium Phosphate Ceramics. ACS Biomater Sci Eng 4: 3347-3355, 2018.

16. Wang XT, Zhou CL, Yan JL, Yan X, Xie HX and Sun CL: The fate of donor osteocytes in fine particulate bone powders during repair of bone defects in experimental rats. Acta Histochem 114: 192-198, 2012

17. Xie H, Ji Y, Tian Q, Wang X, Zhang N, Zhang Y, Xu J, Wang N and Yan J: Autogenous bone particle/titanium fiber composites for bone regeneration in a rabbit radius critical-size defect model. Connect Tissue Res 58: 553-561, 2017.

18. Sun YX, Sun CL, Tian Y, Xu WX, Zhou CL, Xi CY, Yan JL and Wang XT: A comparison of osteocyte bioactivity in fine particulate bone powder grafts vs larger bone grafts in a rat bone repair model. Acta Histochem 116: 1015-1021, 2014

19. Sun C, Tian Y, Xu W, Zhou C, Xie H and Wang X: Development and performance analysis of $\mathrm{Si}-\mathrm{CaP} /$ fine particulate bone powder combined grafts for bone regeneration. Biomed Eng Online 14 47, 2015.

20. Graham N and Qian BZ: Mesenchymal Stromal Cells: Emerging Roles in Bone Metastasis. Int J Mol Sci 19: 1121, 2018.

21. Tajima S, Tobita M, Orbay H, Hyakusoku H and Mizuno H: Direct and indirect effects of a combination of adipose-derived stem cells and platelet-rich plasma on bone regeneration. Tissue Eng Part A 21: 895-905, 2015.

22. Tian Y, Cui L-H, Xiang S-Y, Xu WX, Chen DC, Fu R, Zhou CL, Liu XQ, Wang YF and Wang XT: Osteoblast-oriented differentiation of BMSCs by co-culturing with composite scaffolds constructed using silicon-substituted calcium phosphate, autogenous fine particulate bone powder and alginate in vitro. Oncotarget 8: 88308-88319, 2017.
23. Zhang D, Huang D, Huang Y, Liu Y, Lin B, Yu C, Mou Y, $\mathrm{Wu}$ W, Zhang $\mathrm{H}$ and Lin H: Efficacy of combined therapy of periosteum and bone allograft in a critical-sized defect model in New Zealand white rabbits. Med Sci Monit 20: 2394-2403, 2014

24. Liu F, Chen K, Hou L, Li K, Wang D, Zhang B and Wang X: Determining the critical size of a rabbit rib segmental bone defect model. Regen Biomater 3: 323-328, 2016.

25. Błaszczyk B, Kaspera W, Ficek K, Kajor M, Binkowski M, Stodolak-Zych E, Grajoszek A, Stojko J, Bursig H and Ładziński P: Effects of Polylactide Copolymer Implants and Platelet-Rich Plasma on Bone Regeneration within a Large Calvarial Defect in Sheep. BioMed Res Int 2018: 4120471, 2018.

26. Qi Y, Niu L, Zhao T, Shi Z, Di T, Feng G, Li J and Huang Z: Combining mesenchymal stem cell sheets with platelet-rich plasma gel/calcium phosphate particles: A novel strategy to promote bone regeneration. Stem Cell Res Ther 6: 256, 2015.

27. Guerra I, Morais Branco F, Vasconcelos M, Afonso A, Figueiral H and Zita R: Evaluation of implant osseointegration with different regeneration techniques in the treatment of bone defects around implants: An experimental study in a rabbit model. Clin Oral Implants Res 22: 314-322, 2011.

28. Broggini N, Hofstetter W, Hunziker E, Bosshardt DD, Bornstein MM, Seto I, Weibrich G and Buser D: The influence of PRP on early bone formation in membrane protected defects. A histological and histomorphometric study in the rabbit calvaria. Clin Implant Dent Relat Res 13: 1-12, 2011.

29. Fernandes G and Yang S: Application of platelet-rich plasma with stem cells in bone and periodontal tissue engineering. Bone Res 4: 16036, 2016.

30. Niemeyer P, Fechner K, Milz S, Richter W, Suedkamp NP, Mehlhorn AT, Pearce S and Kasten P: Comparison of mesenchymal stem cells from bone marrow and adipose tissue for bone regeneration in a critical size defect of the sheep tibia and the influence of platelet-rich plasma. Biomaterials 31: 3572-3579, 2010.

31. Lai F, Kakudo N, Morimoto N, Taketani S, Hara T, Ogawa T and Kusumoto K: Platelet-rich plasma enhances the proliferation of human adipose stem cells through multiple signaling pathways. Stem Cell Res Ther 9: 107, 2018.

32. Eda T, Takahashi K, Kanao S, et al: Comparison study between plasma rich in growth factors and platelet-rich plasma for osteoconduction in rat calvaria. J Oral Maxillofac Surg 29: 563-569, 2017.

33. Wang D, Weng Y, Guo S, Zhang Y, Zhou T, Zhang M, Wang L and Ma J: Platelet-rich plasma inhibits RANKL-induced osteoclast differentiation through activation of Wnt pathway during bone remodeling. Int J Mol Med 41: 729-738, 2018

34. Qian Y, Han Q, Chen W, Song J, Zhao X, Ouyang Y, Yuan W and Fan C: Platelet-Rich Plasma Derived Growth Factors Contribute to Stem Cell Differentiation in Musculoskeletal Regeneration. Front Chem 5: 89, 2017

This work is licensed under a Creative Commons Attribution-NonCommercial-NoDerivatives 4.0 International (CC BY-NC-ND 4.0) License. 